\title{
5. Erweiterungen: \\ Zum Programm einer Europäischen Ethnologie
}

Diese Weichenstellungen der siebziger und frühen achtziger Jahre führen schon unmittelbar in die Gegenwart. Nun deren Bild im Sinne eines Überblicks über die Fachlandschaft zu skizzieren ist ein besonders schwieriges Unterfangen, das nur unvollständig und subjektiv ausfallen kann. Es fehlt der klärende zeitliche Abstand, der vielleicht ein abgewogeneres Urteil zuließe. Und es fehlt auch noch manche Klarheit im Fach selbst, dessen Suchbewegungen aus den siebziger Jahren noch keineswegs in einen ruhigen, geraden Kurs gemündet sind. Was einmal Volkskunde war, ist heute in seinen verschiedenen Strömungen und Richtungen so vieldeutig und vielstimmig geworden, daß es sich nur schwer auf einige wenige gemeinsame Nenner bringen läßt. Das deutet sich bereits in der Schwierigkeit an, überhaupt noch eine gemeinsame Fachbezeichnung zu finden, die nicht aus einer mehrgliedrigen Namensaufzählung besteht. Andererseits wurde diese Vielstimmigkeit in den letzten Jahrzehnten auch zu einem produktiven Faktor, so daß man sich viel mehr Einmütigkeit und Ruhe vielleicht gar nicht wünschen sollte. Denn Bewegung verhindert Erstarrung, und Unruhe schützt vor allzu großer Selbstsicherheit.

Bemerkenswert ist auf jeden Fall, daß diese Bewegung der Volkskunde in den siebziger Jahren in mancher Hinsicht offenbar einen deutschen ,Sonderweg“ beschreibt. Während sich etwa in den skandinavischen und in manchen osteuropäischen Ländern oder auch in den Niederlanden eine Europäische Ethnologie neu konstituiert, die sich vor allem aus Anstößen der amerikanischen Anthropologie speist und sich vielfach als regionale „Ethnologie Europas“ versteht, spielt in Deutschland zunächst die Nähe zu einer Erfahrungs- und Alltagsgeschichte eine entscheidende Rolle. Nicht Anthropologisierung, sondern Historisierung der Phänomene steht hier auf der Tagesordnung, um den Raum von Geschichte und Kultur von falschen Mythen und Ideologemen zu befreien - gewiß auch in Reaktion auf die gleichzeitige Auseinandersetzung mit dem Nationalsozialismus. Dafür steht in den achtziger Jahren die Diskussion um die historische Volkskultur, also um einen wesentlichen Selbstverständigungsbegriff der alten Volkskunde, der nun intensiv diskutiert und aus der Sphäre der Ideologie in die Geschichte und Gesellschaft zurückgeholt werden soll: „Volk“ erscheint nicht mehr als bürgerliche Idee gemeinsamen Ursprungs und zugleich als kulturelles, ,fremdes“ Gegenüber in der eigenen Gesellschaft, sondern als eine „heuristische Kategorie“, 
die auf historisierbare und „klassenspezifische“ Erfahrungshorizonte der Vormoderne aufmerksam machen kann (Schindler I984: 54). Auf den weiteren Verlauf dieser aufschlußreichen Debatte brauche ich hier nicht näher einzugehen, er ist vielfach dokumentiert. ${ }^{14}$

So wäre hier eher eine Fachrichtung zu beschreiben, die volkskundliche Blickwinkel verändert und diese in die Perspektiven einer Europäischen Ethnologie erweitert hat, um dadurch ihren Gegenstand, die Kultur, nunmehr jenseits nationaler Grenzen wie wissenschaftlicher Fachgrenzen zu betrachten. Allerdings darf diese Erweiterung keine beliebige Grenzüberschreitung in alle Richtungen und auf jedes Themengebiet bedeuten, sondern sie muß an ein Zentrum aus Erkenntnisinteressen, theoretischen Reflexionen, methodischen Bestimmungen und thematischen Spektren gebunden bleiben, die dem Fach seine ,kognitive Identität“ unter sich rasant verändernden gesellschaftlichen und kulturellen Umständen bewahrt. „Global denken, lokal handeln" gilt in gewisser Weise auch in der heutigen Wissenschaftslandschaft als Maxime.

Dafür scheinen mir vier Positionsbestimmungen wichtig, die ich im folgenden skizzieren will: zum einen die Einbettung der Europäischen Ethnologie in den Kontext der Sozial- und Kulturwissenschaften, zum zweiten die inzwischen neu gewonnenen Sichtweisen und thematischen Kompetenzen, zum dritten die Aufnahme interdisziplinärer wie internationaler Anstöße und zum vierten die Frage, was „Kultur“ im Konzept einer Europäischen Ethnologie bedeuten kann. $\mathrm{Zu}$ alldem sollen die späteren Kapitel dieser Einführung noch ausführlichere Auskünfte geben.

\section{Kontexte}

Was sozial- und kulturwissenschaftliche Erkenntnisinteressen wohl insgesamt verbindet, ist die Grundannahme, daß Gesellschaft stets auf einer bestimmten Vorstellung von sozialer Ordnung beruht. Gefragt wird daher, wie diese Ordnung in unterschiedlichsten Bereichen institutionell und normativ abgesichert ist, welche sozialen und politischen Verhaltensweisen sie hervorbringt, wie sie also ,,vergesellschaftend" wirkt und welche Spielräume sich aus der Differenz zwischen Modell und Praxis für den einzelnen wie für Gruppen in unterschiedlichen Gesellschaften und Epochen ergeben. Dem fügt die kulturwissenschaftliche Beobachtung ihre spezifischen, mehr auf die Praxisseite abzielenden Fragen hinzu: die nach den kulturellen Traditionen und religiösen oder ethnischen Begründungen solcher Ordnungsvorstellungen; nach ihrer alltäglichen Wirkung und Erfahrung in Lebensläufen und Lebenswelten; nach den Bildern, die sich Menschen von dieser Gesellschaft, von deren Teilen, von sich selbst, aber 
auch von anderen Gesellschaften machen, wie also kulturelle „Identitäten" wahrgenommen und ausgehandelt werden; nach der Rolle, die Geschichte und Vergangenheit dabei spielen, danach, wie Menschen und Gesellschaften erinnern, also ein kollektives Gedächtnis und Traditionen aufbauen; oder nach den Symbolen und Ritualen, die zur gesellschaftlichen Verständigung wie zum Konfliktaustragen benutzt werden und die uns letztlich erst eine intersubjektive Wahrnehmung des Anderen ermöglichen.

Begriffsgeschichtlich gesehen, ist die Kultur damit in ihrem Verhältnis zur Gesellschaft in den letzten Jahrzehnten neu bestimmt worden. Kultur meint nicht mehr nur das Traditionale im Sinne von Überlieferung und Brauch oder das Elitäre im Sinne von Bildung und Privileg. Sie versteht sich in ihrer weiten Bedeutung nun vielmehr als die Praxis menschlichen Denkens, Deutens und Handelns, die bestimmten Regeln folgt, diese Regeln ebenso bewahrt wie immer wieder überprüft und verändert und die dadurch Gesellschaft überhaupt erst funktionsfähig macht. Kultur ist somit Medium historischer Prägung wie gegenwärtiger Anwendung, Mittlerin zwischen dem Menschen und seiner Umwelt, zwischen dem Individuum und der Gesellschaft. Sie verkörpert „Führung des Lebens“ und „Deutung des Lebens" zugleich, besteht also in jenem Sinn, den wir den Dingen um uns und unserem Tun geben. Weil sich all diese Bedeutungen eher umschreiben als in einer kurzen Handbuchdefinition fassen lassen, zitiert man heute gern die Metapher von der Kultur als einem ,selbstgesponnenen Bedeutungsgewebe“, die der Kulturanthropologe Clifford Geertz in Anlehnung an Max Weber formuliert hat. Diese Metapher spielt auf das Gemachte und zugleich auf das Komplexe kultureller Praxis an. Und sie ist jedenfalls auch poetisch genug, um das letztlich Ominöse der Kultur, die wir zu sehr selber „sind“", um sie präzise erklären zu können, assoziativ angemessen zu umschreiben.

In den achtziger Jahren wird in den Fachdiskussionen als Hauptaufgabe der Forschung daher immer wieder die „Kulturanalyse“ benannt. Kultur müsse in ihren Praxissystemen und Funktionsweisen untersucht werden; es seien ihre jeweiligen gesellschaftlichen Akteure und Träger zu bestimmen; und sie sei nur in ihrer Wirkung in gesellschaftlichen Prozessen zu erfassen, nicht in statischen Momentaufnahmen. Damit wird - nach der historischen - eine zweite wissenschaftliche Ortsbestimmung vorgenommen, welche die Volkskunde/ Europäische Ethnologie nun auch im Feld sozialwissenschaftlicher Erkenntnisinteressen, Theorien und Methoden positioniert - allerdings mit einem durch die Jahre immer schwerer wiegenden kulturwissenschaftlichen Eigengewicht. Dem strikt sozialwissenschaftlichen Vorgehen, das auf Repräsentativität orientiert ist, auf gesellschaftliche 
Funktionsmodelle, auf deduktive Empirie und Faktorenbestimmung, steht man nach kurzem Liebäugeln dann doch eher skeptisch gegenüber. Vor allem zur engeren Soziologie als der ,Wissenschaft von der Gesellschaft" entsteht ein ambivalentes Verhältnis von Annäherung und Distanz. Einerseits beeindrucken der scheinbar scharfe analytische Blick und die hohe Theoretisierung soziologischer Analysemodelle. Andererseits bleibt Reserve gegenüber den Verlaufs- und Wirkungsmodellen sozialen Handelns oder institutioneller Regelung, die offenbar doch vieles von der Eigengesetzlichkeit sozialer Wahrnehmung und sozialer Praxis durch ihr Interpretationsraster fallen lassen. Auch methodisch nimmt man zwar Elemente soziologischer Datenbeschaffung und -auswertung auf, man arbeitet sie freilich sehr oft um für den eigenen Gebrauch, in dem die Methoden dann eher ,gemixt“ angewandt werden - für die einen ein ,Sakrileg“, für die anderen ein Indiz für ,Kreativität“.

Als grundlegend für das methodische Selbstverständnis erweist sich aber doch der ,,hermeneutische“, also der deutende und interpretierende Zugang zu den Quellen und Phänomenen. Hermann Bausinger hat diese „Spezifik volkskundlicher Arbeit“ einmal so beschrieben: „Insgesamt also scheint die Anwendung, weicher" Instrumente charakteristisch $\mathrm{zu}$ sein, wobei weich nicht schwach bedeutet, sondern eher behutsam, anschmiegsam." Er weist freilich darauf hin, daß diese ,weichere Methodik ihre vollen Möglichkeiten oft erst entfaltet in Verbindung mit den durch ,harte' Methoden gewonnenen Vorgaben", daß aber doch plausible Argumente letztlich den weichen Methoden Vorrang einräumen. „Diese sind, zumindest auf den ersten Blick, weniger, exakt'; sie sind nicht imstande, präzise abgegrenzte Kategorien und Datenmengen bereitzustellen. Aber sie erweisen sich manchmal auf den zweiten Blick als ,genauer", als wirklichkeitsadäquater.“ Denn: „Die an harten Daten oft gerühmte klare kategoriale Zuordnung und damit Vergleichbarkeit hat ihre negative Seite: sie operiert oft mit Eindeutigkeiten, die so nicht vorliegen, und sie läuft immer Gefahr, die Zwischenbereiche und Zwischentöne zu ignorieren. (...) Die implizite Zielsetzung der ,exakten“ Methodik, zu möglichst präzisen Bestimmungen des jeweiligen Durchschnitts zu gelangen, droht dazu zu führen, daß die breiten Ränder zugunsten einer manchmal eher imaginären Mitte vernachlässigt werden; an die Stelle der - nicht ungefährlichen - Anekdotik der Einzelbeobachtung kann, zugespitzt gesagt, eine Anekdotik der Mittelwerte treten.“ Und schließlich: „Die Verwendung weicherer Methoden erlaubt es dem Forscher wahrscheinlich doch eher, das Forschungsinteresse und das Interesse der Erforschten wenn nicht zur Deckung zu bringen, so doch einander anzunähern. (...) Eben dies aber scheint mir eher möglich zu sein, wenn auch die Erhebungsme- 
thoden , dialogische' sind, wenn sie eine gewisse Symmetrie erlauben, wenn also das Forschungsdesign auf die besonderen Zusammenhänge und Lebensumstände Rücksicht nimmt." (Bausinger I980: i8ff.) Was damit im Extremfall gemeint ist, mag ein - vereinfachendes - Beispiel aus der historischen Demographie verdeutlichen: Dort läßt sich mit Durchschnittszahlen etwa zur Lebenserwartung von Menschen im I7. oder I 8. Jahrhundert zwar trefflich statistisch operieren, allerdings keine Vorstellung davon gewinnen, wie alt die Menschen damals wirklich wurden. Angesichts von hoher Kindersterblichkeit einerseits und doch erstaunlich langen Lebensläufen andererseits beschreibt eine Zahl von 43 Jahren als durchschnittlicher Lebenserwartung eigentlich das Gegenteil der historischen Erfahrung. Man starb ausgesprochen selten im 43. Lebensjahr, vielmehr entweder sehr jung oder doch auch für unsere Begriffe relativ alt.

Ein anderer, wesentlicher Unterschied zur Soziologie besteht in diesen achtziger Jahren darin, daß viele ethnologische Methoden vor allem „,im Feld“ entwickelt und angewendet werden müssen. Durch die stärkere Orientierung auf gegenwartsbezogene Themen wird die „Teilnehmende Beobachtung“ am Ort gerade von der jüngeren Forschergeneration aus der (ehemaligen) Volkskunde immer häufiger praktiziert. Damit wird Forschung auch als eigene kulturelle Praxis sichtbar, freilich auch zum Problem, weil dadurch neue methodologische Fragen entstehen. So stellen sich hier neue Nähen vor allem zur Völkerkunde her, die sich in einem strikten Verständnis mit gutem Recht „im Feld“ sehr viel heimischer fühlen kann. Aber auch die Bezüge zur Ethnopsychoanalyse wie zur Psychologie verstärken sich, die teilweise ebenfalls über lange Erfahrungen und eigene methodische Instrumentarien der wissenschaftlich kontrollierten „Begegnung mit dem Anderen“, also etwa der Feldforschung und des Interviews, verfügen.

$\mathrm{Zu}$ einem entscheidenden Punkt der wissenschaftlichen Ortsbestimmung wird schließlich die seit den siebziger Jahren so intensiv diskutierte Frage nach der Wissenschaft als sozialer und politischer Praxis. Manche Euphorie dieser Jahre ist verflogen. Das Feld hat sich oft als eigensinniger herausgestellt denn erwartet. Und die Aufklärungs- und Eingriffsmöglichkeiten der Wissenschaft erscheinen nach mancher Enttäuschung doch eher begrenzt. Dennoch bleibt die knappe Frage: „Wem nützt Volkskunde?“ (D. Kramer 1970) ein steter Stachel, der neben ethischen und moralischen Forschungsproblemen immer wieder auch auf grundsätzliche erkenntnistheoretische wie gesellschaftspolitische Dimensionen ethnologischer Forschung verweist. Auf offene Fragen, die in den neunziger Jahren wieder aufgenommen, nun jedoch nicht mehr von den Sozialwissenschaften insgesamt, sondern vor allem von der Linguistik, aber auch der Kultur- 
anthropologie und Ethnologie selbst aufgeworfen und als „Krise der Repräsentationen“ diskutiert werden: Wer forscht, wer schreibt über wen, für wen? Mit dieser Neubestimmung der Kulturforschung als einer sozialen Praxis der kulturellen Begegnung - im Feld wie in der Öffentlichkeit - wird nunmehr eine erkenntnistheoretische Position bezogen, die sich „kulturwissenschaftlich“ verselbständigt und plötzlich eine ungeahnte Anziehungskraft auf andere Disziplinen ausübt. Nicht nur zur Freude der Ethnowissenschaften, die sich unversehens im Kreise lauter neu entdeckter „Kulturwissenschaften“ wiederfinden.

\section{Neue Sichtweisen}

Angestoßen vor allem durch die Frage nach dem gesellschaftspolitischen Standort des Faches, wurde seit den siebziger Jahren immer wieder nach neuen Modellen und Ordnungsvorstellungen gesucht, die uns den geschichtlichen Horizont, den gesellschaftlichen Raum und seine kulturelle Landschaft anders, besser, verständlicher im Blick auf die darin lebenden Menschen zu erklären vermögen. Dafür sind in der Tat neue Perspektiven gefunden worden, die jedoch unverkennbar nicht allein im Wissenschaftsraum geprägt, sondern die in hohem Maße auch durch gesellschaftliche Bewegungen und politische Veränderungen geschaffen werden. Wissenschaft ist weniger denn je ein von der Gesellschaft abgetrennter, durch Weihe und Tabu geschützter Raum. Es gibt heute kein ergriffenes Publikum mehr, das ehrfürchtig wissenschaftliche Zeremonien beobachtet und neuen Erkenntnissen lauscht. Wissenschaftliche und gesellschaftliche Debatten verschränken sich statt dessen ineinander und in einer Weise, die der Wissenschaft keine privilegierte Position mehr außeroder oberhalb des gesellschaftlichen „Diskursuniversums“ einräumt, sondern sie in dessen Wissens- und Alltagsordnungen einbezieht.

Dies gilt in besonderem Maße für eine Wissenschaft, die sich für die Dinge des Alltags zuständig erklärt, also für jene Gesellschaftsund Lebensbereiche, in denen jeder als „Alltagsmensch“ über eigene Wahrnehmungen, eigenes Wissen und eigenes Expertentum zu verfügen scheint. Gerade hier gibt die Forschung nicht einfach mehr die Begrifflichkeiten der Welt als Wirklichkeit vor, sondern sie reagiert in mancher Hinsicht nur noch auf gesellschaftliche Modi der Wahrnehmung und des Begreifens, die als „Weltbilder“ im vorwissenschaftlichen Bereich der Lebenswelten, der Medien und der politischen Diskussionen entstehen.

Um dafür einige Bespiele aus unseren Feldern zu nennen: Die Wendung hin zu einer Alltags- und Erfahrungsgeschichte war eben nicht allein Resultat geschichtswissenschaftlicher und volkskundli- 
cher Wissenschaftsdebatten, vielmehr in hohem Maße auch einer internationalen Laienbewegung, die seit den siebziger Jahren unter Alltagsgeschichte nicht nur das Wissen um die Lebensumstände und Lebenswege der „einfachen Leute“ in der Vergangenheit verstand, sondern ebenso die Beschäftigung mit Geschichte im Sinne der Erhellung der eigenen Vorgeschichte: Geschichte der eigenen Stadt, der eigenen Berufsgruppe, auch der eigenen Familie. Ebensowenig läßt sich die Entwicklung einer ökologischen Perspektive allein aus den Bemühungen von natur- und geisteswissenschaftlichen Kritikern des Zivilisations- und Fortschrittskonzepts erklären. Auch hier waren und sind die ökologischen und ,grünen“ Bewegungen mitverantwortlich dafür, daß neue Fragen und Sensibilitäten im Blick auf das Verhältnis Mensch-Natur entstehen. Und auch die Wahrnehmung der grundsätzlichen Bedeutung der Geschlechterfrage ist weniger die Folge wissenschaftlicher Erklärungsmodelle als vielmehr das Ergebnis der politischen Frauenbewegung, die einer männerzentrierten gesellschaftlichen Kultur den Kampf ansagte und damit zugleich unsere Geschichtsbilder umschrieb. Ungleichheit im doppelten Sinne von Verschiedenartigkeit wie von Privilegierung bzw. Unterprivilegierung hat dadurch völlig neue Bedeutungen erhalten. Das gilt schließlich sogar für den Bereich der ethnischen Fremdbilder, die weniger durch philosophische Gedanken als vielmehr durch gesellschaftspolitische Diskussionen um die multikulturelle Gesellschaft und durch „Wortmeldungen“ von Migrantengruppen oder postkolonialer Länder der „Dritten Welt“ als Machtmittel entlarvt, freilich auch oft selbst benutzt werden. Daß die Ethnowissenschaften in vielen dieser Felder frühzeitig Partei ergriffen haben, schafft noch kein besonderes Verdienst, aber es eröffnet die Chance auf die „reflexive“ Auffassung unserer Wissenschaften.

Das sind nur einige wichtige Beispiele für generelle Perspektivenänderungen, die gesellschaftlich angestoßen sind und in der Wissenschaft bewirken, daß deren Fragen an Gesellschaft und Kultur anders gestellt werden und die Antworten darauf wesentlich komplizierter ausfallen. Denn die Erklärungskoordinaten des Alltags, der Umwelt, der Geschlechter, der ethnischen Identitätskonstruktionen in jedes Untersuchungskonzept einer Lebenswelt einzubeziehen bedeutet zugleich, immer wieder unterschiedliche Antworten auf dieselbe Frage zu erhalten. Die Wahrnehmungen der Gegenwart und die Erklärungen der Geschichte sind in dem Maße vielstimmig geworden, in dem wir erkennen, daß die Aufgabe von Wissenschaft wie von Alltagskultur nicht darin besteht, homogene Gesellschaften und homogene Bilder zu organisieren, sondern im Gegenteil dazu fähig zu machen, mit Unterschieden und Verschiedenheit umzugehen (Hannerz I992: I4). Der französische Ethnologe Marc Augé konstatiert: „Viel- 
mehr verlangt die heutige Welt aufgrund ihres beschleunigten Wandels selbst nach dem anthropologischen Blick, das heißt: nach einem neuartigen und methodischen Nachdenken über die Kategorie der Andersheit." (Augé 1994: 32)

Diese Entwicklung schlägt sich deutlich in den Forschungsrichtungen nieder, die sich in den achtziger Jahren entwickeln und zum Teil das Fach bis heute mit prägen. Dabei geht es etwa um Alltagsgeschichte und historische Mentalitäten, um Vorstellungen also vom Denken, Fühlen und Handeln von Menschen in anderen Epochen und mit anderer „Weltanschauung“; um soziale und geschlechtliche Ausprägungen lebensgeschichtlicher Erfahrung, wie sie die Oral History in Frauen- und Männerlebensgeschichten untersucht; um urbane Lebensstile und deren historische wie postmoderne Signaturen im Rahmen stadtethnologischer Forschungen; um Fragen der kulturellen Repräsentation von Einwanderergruppen und der multikulturellen Politik von Einwanderungsländern im Zusammenhang von Migrationsprozessen; um geschichtliche Entwicklungen der Reisekultur wie um Erfahrungen und Wirkungen des modernen Tourismus bei den Reisenden wie den Bereisten; um Musik-, Kleidungsstile und andere ästhetische Praxen in Jugendkulturen sowie in Freizeitkulturen; um die Bedeutung von Bildern und Medien für die Entwicklung unserer alltäglichen Wahrnehmungsgewohnheiten im Sinne einer visuellen Anthroplogie. Und es geht in besonderer Weise um Fragen der eigenen Wissenschaftsgeschichte und der Forschungsmethodik, die kritisch beleuchtet werden im Blick auf ihre inhaltlichen Ergebnisse wie auf ihre gesellschaftlichen Auswirkungen.

\section{Blicke von draußen und nach draußen}

All diese Felder liegen auch im Schnittpunkt internationaler Forschungen. Die Vorstellung einer volkskundlichen Erforschung der eigenen Kultur, die zuvor immer vertraut und nah erschien, hat sich mit der Erkenntnis, daß Wahrnehmungen des „Andersseins“ und der „Fremdheit" wenig mit nationalen Grenzen zu tun haben, zu einer ethnographischen Forschung auch in anderen europäischen Regionen erweitert. So wird das Leben an der Grenze auch in Frankreich untersucht, Tourismus in Sizilien, magisches Denken in Norditalien, Bauernleben in Irland, Aussteigerbiographien in Griechenland.

Zugleich sind es umgekehrt auch Anstöße von ,draußen“ in Gestalt internationaler Leitbegriffe und Forschungstheorien, welche die Interessen und die Blicke in neue Richtungen lenken. Diese Anstöße kommen im Fall der deutschen Volkskunde zunächst nicht - wie man heute vielleicht erwarten könnte und wie in anderen europäischen Ländern bzw. Schwesterfächern geschehen - aus der amerika- 
nischen Anthropologie. Vielmehr steht die Zuwendung etwa zu plebejischen und volkskulturellen Traditionen der vorindustriellen Zeit deutlich unter dem Einfluß geschichtswissenschaftlicher Diskussionen insbesondere in England um einen weiten Kultur- und einen neuen marxistischen Klassenbegriff, verbunden mit den Namen des Historikers Edward P. Thompson (1987) und des Soziologen Raymond Williams (1977). Über schichtspezifische Jugendkulturen und deren besondere ästhetische Stile wird im Gefolge der Cultural Studies des Birminghamer Center for Contemporary Cultural Studies geforscht, das „Kultur“" in diesem Feld als einen besonders wirksamen sozialen Integrations- wie als Abgrenzungsmechanismus jugendlicher peergroups beschreibt (Clarke u.a. I979). Der Beginn einer Mentalitätengeschichte in Deutschland ist ohne das französische Vorbild der Histoire des Mentalités nicht denkbar, in der den Kontinuitäten, der longue durée von Denkweisen, Werten und Ritualen im Sinne langer kultureller Traditionen von der Neuzeit bis in die Moderne nachgegangen wird; sie ist verbunden mit Namen wie Fernand Braudel (I97I), Emmanuel LeRoy Ladurie (I983) oder auch dem des italienischen Historikers Carlo Ginzburg (1993a/b), der Alltags- und Mentalitätengeschichte auf neue Weise zu einer „Mikrogeschichte“ der einzelnen Individuen und Orte verknüpft. Erst dann, etwa durch die intensivere Beschäftigung mit Migrantenkulturen in Europa oder mit Fragen der „Kultur der großen Stadt“, wie sie eine Stadtethnologie und -anthropologie stellt, wirken auch stärker die Vorbilder der USamerikanischen Cultural Anthropology und Stadtsoziologie, die neue Wege zur Erforschung des „,nahen Anderen“ einschlagen (Lindner 1990). In diesen international diskutierten und begangenen Feldern treffen sich im übrigen nun wieder manche der lange Zeit getrennt verlaufenen Wege der ehemaligen Volkskunde und der Völkerkunde.

Damit ist deutlich geworden, welch großen Einfluß auch interdisziplinäre Perspektiven auf die Neuverortung des Faches haben. Man kann dies wieder an den eben genannten Beispielen festmachen: Die Beschäftigung etwa mit der historischen Volkskultur und einer Mentalitätengeschichte erfolgt gleichsam als Reaktion auf deren „Entdeckung“ durch die Historiker, ironischerweise kurz nachdem die Volkskunde das „Volksleben“ gerade verlassen hatte. In der neuen gemeinsamen Beschäftigung mit dem Leben vorindustrieller Unterschichten und deren Praktiken wird nun freilich genau historisiert, in Mikrostudien detailliert geforscht, quellenkritisch betrachtet, so daß „Nahaufnahmen“ geschichtlicher Lebenswelten entstehen jenseits der Mythen und Idyllen. Daß dabei wie im Rahmen der Alltagsgeschichtsschreibung zeitweise wohl auch eine „Geschichte der Entrechteten“ mit moralischem Anspruch und im Ein- 
zelfall auch mit sozialromantischer Einfärbung geschrieben wird, schmälert insgesamt nicht den Wert der dabei gewonnenen Erkenntnisse. Ähnliche interdisziplinäre Kooperationen ergeben sich auch im Bereich der Geschlechtergeschichte oder der Religiositätsforschung. Von der Soziologie wiederum kommen Anregungen, die früher rein historisch betriebene volkskundliche Tradition der Kleidungs- und Nahrungsforschung zu erweitern auf eine Lebensstilforschung, die nach der Entwicklung von Konsum- und Geschmacksmustern, von Gesellungs- und Freizeitformen, von Schicht- und Gruppenkulturen bis in die Gegenwart fragt. Wesentliche Impulse dazu gibt der französische Kulturforscher Pierre Bourdieu, der bezeichnenderweise Ethnologe und Kultursoziologe zugleich ist und in seinen Arbeiten zur ,sozialen Distinktion“ jene Mechanismen deutlich macht, die als kulturelle Praktiken zur sozialen Abgrenzung zwischen verschiedenen Gesellschaftsgruppen dienen und sich in Bildungsstrategien und Geschmackspräferenzen ausdrücken. Er entziffert damit die symbolische Grammatik eines „Raumes der Lebensstile“, in dem Gruppenzugehörigkeit und sozialer Status über die kleinen symbolischen Gesten und kulturellen Muster des Alltags bestimmt werden. ${ }^{15}$

Man kann auch die Literaturwissenschaft und die Germanistik anführen, die etwa durch ihre Forschungen zur Reiseliteratur wertvolle Hinweise gegeben haben zur Lesart „fremder“ Texte oder die sich im Bereich autobiographischer Dokumente auch mit populären Erinnerungsformen auseinandersetzen (Warneken I985). Man wird die Sprachwissenschaft nennen müssen, die mit ihren linguistischen und semiotischen Modellen wesentlich dazu beigetragen hat, Kultur in ihrer ,grammatikalischen“ Struktur und in ihrer Zeichenhaftigkeit besser zu verstehen. Gleiches gilt für die Psychologie, deren methodologische Warnungen vor einer allzu naiven ethnologischen Feldforschung geholfen haben, daß ethnopsychoanalytische Formen einer begleitenden „Beobachtung der Beobachtung“ uns allmählich verständlich machen, wie kompliziert sich der kulturelle Kontakt zwischen Beforschten und Forschenden gestaltet und wie groß die $\mathrm{Ge}-$ fahr ist, eigene Vorstellungen und Verstehensweisen auf „,den Anderen" zu projizieren (Erdheim I982; Nadig 1982). Und schließlich ist selbstverständlich die in außereuropäischen Feldern arbeitende Ethnologie zu nennen, zu der die Verbindungen in den achtziger Jahren wieder wesentlich enger geworden sind und mit der sowohl theoretische Fragen zum Kulturverständnis als auch methodische Vorgehensweisen in der Feldforschung ausgetauscht werden (Wolf I986). Der Gedanke der Feldforschung in einem strengeren Sinne ist ja in vieler Hinsicht erst von den außereuropäischen in die europäischen Beobachtungsräume übernommen worden. 
Dies sind nur einige wenige Beispiele für jene interdisziplinären wie internationalen Erweiterungen der Perspektive, die maßgeblich dazu beigetragen haben, daß sich im Fach Volkskunde ein anderes, neues Verständnis, eben das einer Europäischen Ethnologie, entwikkelt hat. Umgekehrt strahlte diese Fachentwicklung auch auf die anderen Disziplinen aus, gab ihnen wiederum neue Impulse. Vieles davon scheint so naheliegend und sich so organisch zu vollziehen, daß es im Fluß der Entwicklung heute als etwas Selbstverständliches wahrgenommen wird. Doch wer eine volkskundliche Studie etwa der sechziger Jahre neben eine europäisch-ethnologische Studie von heute legt, wird vielfältige Unterschiede in der Gegenstandsauffassung, in der theoretischen und methodischen Anlage wie auch in der Erklärung des Erkenntnisinteresses nicht übersehen können: Es handelt sich bei der Europäischen Ethnologie nicht nur um ein „,geliftetes" Modell der Volkskunde.

Was eine so veränderte Fachkonzeption bedeuten kann, ist damit von ihren Rändern und von ihren Schnittpunkten mit anderen Disziplinen her angedeutet worden. Ich will nun versuchen, auch ihr eigenes Selbstverständnis in Stichworten zu skizzieren, die aber nicht mehr sein sollen als eine Hinführung auf die nachfolgenden ausführlicheren Versuche einer Programm- und Standortbestimmung.

\section{Selbstverständigungen}

Die klassische Unterscheidung zwischen der Volkskunde und der Völkerkunde wurde lange Zeit in Form einer einfachen Gegenüberstellung bestimmt, wonach sich die Volkskunde mit der ,eigenen Kultur“ beschäftige, die Völkerkunde hingegen mit ,fremden Kulturen“. Als Metapher wird diese Formulierung auch heute noch gerne benutzt, weil sie griffig und plastisch erscheint. Freilich beinhaltet schon diese kurze Formel eine Reihe von Vorannahmen, denen wir heute nicht mehr ohne weiteres folgen können. Die ,eigene Kultur", die meist innerhalb sprachlicher und politisch-nationaler Grenzen gedacht war, hat sich nämlich in ihrer sozialen Gliederung, in ihrer Einbindung in globale Kulturprozesse und eben auch in der Begegnung der forschenden Wissenschaftskultur mit den beforschten Gruppenkulturen als nichts weniger denn homogen, vertraut, also „eigen“ erwiesen. Zwar finden sich stets übergreifende, scheinbar gemeinsame Züge, aber bei genauerem Hinsehen erweist sich dieses Verstehen der anderen ,im Eigenen“ als sehr viel komplizierter: Auch vieles Nahe bleibt uns fremd. Umgekehrt gilt für ,das Frem$d$ “, daß uns dort vieles vertraut scheinen mag, wenn wir einmal die Vorurteile überwunden haben, daß große geographische Entfernung, 
andere Hautfarbe, andere Sprache, andere Religion automatisch „Fremdheit" bedeuten müssen.

Daher muß das Anliegen einer Europäischen Ethnologie heute doch anders formuliert werden. In eine - ungeliebte - Kurzformel gebracht, könnte es als die Beobachtung und Erforschung des Anderen in der Kultur beschrieben werden, wobei dieses Andere stets durch unseren forschenden Blick fixiert und konstruiert wird in dem Moment, in dem wir es zum Beobachtungsgegenstand machen: Menschen, Gruppen, Verhaltensweisen, Wertehorizonte, Symbole, Dinge. Nichts ist zunächst ,anders“, sondern es wird dazu, indem wir es ins Auge fassen, uns ihm annähern, es zu verstehen versuchen. „Verstehen“ bedeutet ja lediglich, daß wir es in unser Deutungssystem, in unsere kulturelle Sprache übersetzen wollen, um zu begreifen, was und wie es für uns ist - und gleichzeitig, um zu begreifen, wer und wie wir sind, indem wir eben wiederum, ,anders“ sind. Die jüngeren Diskussionen in der amerikanischen Kulturanthropologie um das „Eigene“ und das „Fremde“ bzw. um theoretische wie methodologische Probleme des „Verstehens“ haben bei dieser Erkenntnis gewiß eine wichtige Rolle gespielt (Clifford/Marcus I986). Und unumkehrbar ist die Erkenntnis, daß diese Verstehens- und Repräsentationsprobleme alltägliches und wissenschaftliches Denken gleichermaßen betreffen, daß auch „wissenschaftliches Wissen“ durch „soziales Handeln produziert“" wird (Welz I996: 28).

Daraus ergeben sich einige wesentliche Bestimmungslinien für unser Kulturverständnis. Kultur kann danach weder als ein festes System von Traditionen, Werten, Handlungsmustern und Symbolen verstanden werden, das sich in beständiger Wiederholung fortsetzt, noch kann sie gewissermaßen in Landkartenform gebracht werden, die politisch-geographisch-sprachliche Einheiten einfach als „,nationale Kulturen“" deklariert. Kultur meint vielmehr den ständigen Prozeß des praktischen Aushandelns jener Regeln, nach denen Menschen, Gruppen und Gesellschaften miteinander verkehren, nach denen sie sich untereinander verständigen wie gegenseitig abgrenzen. Damit sind immer Regeln und Verhaltensmuster ganz unterschiedlicher Reichweite und Geltungsdauer gemeint: universell wie situativ gültige, historisch überlieferte wie aktuell erworbene, bewußte wie unbewußte, kollektive wie individuelle. Solche Kultur meint Gesellschaft im Vollzug ihres praktischen Lebens.

Für eine Europäische Ethnologie scheint mir dies zu bedeuten, daß sie Kultur zuallererst als alltägliche Praxis verstehen muß, als ein Ineinander von Verhaltensregeln, Repräsentationsformen und Handlungsweisen in konkreten sozialen Kontexten, eng an die Menschen als Akteure gebunden und nicht in einem über ihnen schwebenden Wertehimmel vermutet. Wie Menschen ihr Zusammenleben organi- 
sieren, welches Verhältnis zu sozialer Umwelt und Natur eingegangen wird und welches Bild sich die Menschen von diesen Beziehungen selbst machen - diese scheinbar so einfachen Fragen nach der Alltagskultur müssen im Vordergrund stehen. Dabei spielt auch die räumliche und ethnische Strukturierung der Kultur gewiß eine Rolle, aber eben nicht im Sinne einer kulturellen Volks- und Länderkunde. Der Hinweis auf das Ethnische, der in der Fachbezeichnung zentral enthalten ist, fordert vielmehr dazu auf, sich kritisch mit der Frage auseinanderzusetzen, weshalb und in welchen Situationen die Vorstellung einer ethnischen oder nationalen Kulturgemeinschaft benutzt wird, um Selbstbilder und Fremdbilder zu entwerfen. Und stets muß im Hinterkopf bewußt bleiben, daß die Ethnowissenschaften in der Vergangenheit nicht unwesentlich daran beteiligt waren, dieses „ethnische Paradigma“ als ein vermeintlich festes Grundschema kultureller Herkunft und Zugehörigkeit in den Köpfen der Menschen zu verankern.

Es geht also um Kultur als Praxis nicht nur im Sinne vordergründigen Handelns, sondern auch in dem von Vorstellungen und Deutungen, von Urteilen und Vorurteilen. Und diese Tendenz, möglichst alle Phänomene, mit denen Menschen konfrontiert werden, in ein System von Wahrnehmungen und Bildern zu bringen, durch die sich die Welt „ordnen“ läßt, begegnet uns in allen Forschungsfeldern: im Zusammenhang der Migration wie des Reisens, der Folklore wie der Mode, der Freizeit wie der Eßkultur. Die Bildung kultureller Stereotype ist gleichsam ein universelles ,interkulturelles“ Muster, das Orientierung durch Vereinfachung, durch Komplexitätsreduktion, durch Klischeebildung ermöglicht. Ethnologie hat vor allem diese Funktion des kulturellen Deutens und Verstehens in Lebenswelten und Alltagshorizonten zu untersuchen.

Nun begründet dies noch nicht, weshalb eine solche Ethnologie im europäischen Rahmen betrieben werden soll. Eher scheint dies dem Ziel grenzüberschreitenden Denkens und Forschens zu widersprechen. Wenn man sich nicht mehr wie die Volkskunde an die deutsch(sprachig)en Grenzen des Forschungsfeldes halten mag, weshalb dann diese erneute, lediglich räumlich erweiterte Begrenzung?

\section{Europäisches Denken?}

In der Tat bedeutet dieser Rahmen sicherlich mehr eine Setzung, mehr eine pragmatisch als grundsätzlich zu begründende Perspektive. Und natürlich soll damit keineswegs jene eurozentrische Perspektive neu festgeschrieben werden, die wir gegenwärtig in so vielen wissenschaftlichen wie politischen Zusammenhängen kritisieren. Der Blick darf also nicht an den Grenzen Europas enden, er muß im Gegenteil 
europäische Entwicklungen in globalen Zusammenhängen sehen, er muß vergleichen, und er muß vor allem die Blicke ,von draußen“" auf Europa sensibel registrieren. Aber es gibt auch eine Reihe von kultur- und wissenschaftsgeschichtlichen Sachverhalten, die sinnvolle Argumente dafür liefern, weshalb die Möglichkeiten und Fähigkeiten ethnologischen Forschens sich in einem europäischen Horizontausschnitt besonders entfalten können. Dazu ein kurzer Rekurs in die Geschichte.

Spätestens seit der Renaissance folgt geschichtliches und gesellschaftliches Denken in Begriffen von Kultur, Politik, Religion einem Bild und Modell „,europäischer Zivilisation“. Damit sind Abgrenzungen gezogen gegen jene „fremde“ und „exotische“ Welt draußen, die man entdeckt hat und nun kennenlernen will. Dazu gehört aber auch bereits die Entwicklung der Vorstellung von nationalen Staaten und Kulturen, wie sie etwa in Spanien, England oder Frankreich bald erste Gestalt erhalten. Mit dieser Trias von Gesellschaft, Staat und Nation beschäftigen sich die Philosophie, die Geschichtswissenschaft, die Nationalökonomie, die Archäologie, und es stoßen im späten I 8. Jahrhundert die Vorläufer der Volks- und Völkerkunde hinzu. In diesem europäischen Weltbild ist schon früh ein Leitmotiv enthalten, welches das „Nachdenken“ über die eigene wie über andere Gesellschaften auf die Tagesordnung setzt. Es konstituiert sich die Gesellschaft, die sich selbst genau beobachtet, die ein Bild von sich und anderen entwirft, die vergleicht, die damit Antworten sucht auf die Fragen: Wer sind wir? Wo stehen wir? Worin sind wir anders als andere?

Diese Form der ständigen gesellschaftlichen Selbstbeobachtung und Selbstthematisierung verkörpert ein europäisches Spezifikum, das sich in anderen Kontinenten und Kulturen offenbar nicht wiederfindet - jedenfalls in solcher Ausprägung und Kontinuität. In der europäischen Moderne wird dieser Gedanke noch verstärkt, wenn nach rationalen Kriterien und Modellen gesucht wird, in denen sich „Fortschritt“ und „Zivilisation“ messen lassen. Selbstreflexivität als ein beständiges öffentliches Nachdenken über die eigenen Grundlagen und Entwicklungen, als ein beständiger konkurrierender Vergleich mit den Entwicklungswegen der anderen wird so zu einem Signum der europäischen Wissens- und Wissenschaftskulturen (Wolf I986). Und dieses Signum scheint noch heute eingewachsen in unsere Denksysteme, in die uns medial vermittelte Welt, in unsere Bilder von gesellschaftlicher Identität.

Dieses europäische Denken hat einerseits den „kolonialen“ und „fremden“ Blick mit entwickelt, der das Verstehen des Anderen in Geschichte wie Gegenwart so schwierig macht. Andererseits hat sich daraus ein Repertoire kultureller Repräsentationsformen und Wahr- 
nehmungstechniken entwickelt, eine auch wissenschaftlich begründete Kultur der Beobachtung und Begegnung, die interkulturellen Regeln folgt und interkulturelle Verständigungsprozesse ermöglicht. Selbst- und Fremdcharakterisierungen etwa im Bereich der Geographie, der Wirtschaft, der Politik, der Kulturgeschichte, auch der Alltagskultur und der Mentalitäten folgen im europäischen Rahmen relativ festen Indikatoren und Mustern, die in gewisser Weise eine Übersetzung jener wechselseitigen Bilder ermöglichen, die sich europäische Gesellschaften voneinander machen. Die Reiseliteratur und Reisekultur der vergangenen drei Jahrhunderte bietet dafür eine einzigartige Kette von Dokumenten und Belegen.

Es ist also ein komplizierter Prozeß der wechselseitigen Konstruktion und Projektion von Bildern entstanden, in dem alle Beobachtende und Beobachtete zugleich sind und in dem die Möglichkeiten des „Verstehens“ und die Gefahren des „Mißverstehens“ vielleicht sogar zu einer gewissen Balance finden. Europa als diesen historischen Raum eines eingeübten Umgangs mit kulturellen „Weltbildern“ ernst zu nehmen, es also weniger als eine „Kulturen-Landschaft" denn als großen Diskursraum , des Kulturellen“ zu begreifen, es zugleich als zivilisationsgeschichtliche „Werkstatt des ethnologischen Blicks" und damit eines spezifischen kulturellen wie wissenschaftlichen Praxismusters zu verstehen, dies alles scheinen mir kultur- wie wissenschaftsgeschichtlich plausible Argumente für ein Wissenschaftskonzept „Europäische Ethnologie“ zu sein.

Nicht zuletzt folgen diese Überlegungen der in den letzten Jahren gewonnenen Erkenntnis, wonach das „Beobachten unseres Beobachtens" eine Hauptaufgabe ethnologischer Wissenschaft sein muß. Diese Erkenntnis hat sich parallel zur Volkskunde auch längst in der Völkerkunde durchgesetzt, in der ,Fremdheit als methodisches Prinzip“ bereits sehr viel früher erkannt wurde. Inzwischen wird es auch dort als eine zentrale Herausforderung betrachtet, sich mit Methoden, die in der ,kulturellen Fremde“ entwickelt wurden, der europäischen Kultur anzunehmen. Nachdem die Verbindung zwischen beiden Fächern lange Zeit nur auf der Ebene verwandter Forschungsmethoden gesehen wurde, deuten sich hier überdies stärkere theoretische und perspektivische Annäherungen an. Bereits 1982 skizziert ein Sammelband mit dem Titel (Europäische Ethnologie» (Hauschild/Nixdorf I982) einen solchen Weg in eine europäische „Inlandsethnologie“. Zugleich wird in diesen Skizzen aber wie in neueren völkerkundlichen Forschungen innerhalb Europas deutlich, daß sich bei aller Annäherung die „Handschriften“ noch längst nicht gleichen, daß die theoretischen und methodischen Zugänge wie die kognitive Identität beider Fächer nach wie vor deutliche Unterschiede aufweisen (Kokot/Dracklé I996). Diese Differenz ist ein wichtiger 
und keineswegs negativer Befund, denn wie für die gesellschaftlichen gilt auch für die Wissenschaftskulturen, daß gerade aus der bewußten Begegnung ,des Eigenen“ mit „dem Anderen“ erst eine Erkenntnis entsteht.

Schließlich trägt diese europäische Dimension einer Entwicklung Rechnung, die sich in den letzten Jahrzehnten in den traditionellen Berufsfeldern von VolkskundlerInnen vollzogen hat. In den vielfältigen Berufsbereichen vom Museum bis zur Universität, vom Kulturjournalismus bis zu Weiterbildungsinstitutionen, von der Kulturpolitik bis zum Kulturmanagement ist ein Zuschnitt fachlicher Expertise gefragt, der über enge regionale und nationale Zäune hinausweist. Denn die Berufsfelder erstrecken sich in gesellschaftliche Bereiche, in denen europäisches und globales Denken längst keine wohlfeile Floskel mehr ist, sondern konkrete Begegnungsformen und alltägliche Wissensordnungen beschreibt. In Stadtverwaltungen, Kultureinrichtungen, Medien, Forschungsinstituten ist die Kultur längst vielsprachig geworden. Und selbst im Museum, das für das Vergangene zuständig ist, kann eine „Deutsche Volkskunde“ in ihrem buchstäblichen Wortsinn heute nurmehr Ausstellungsobjekt sein. Europäische Ethnologie will und soll insofern auch einen praxisbezogenen europäischen Horizont ethnologischen Arbeitens beschreiben. Sie kann dies mit dem Anspruch und in dem Selbstbewußtsein tun, die kulturellen wie politischen Entwicklungen in unseren Gegenwartsgesellschaften mit kritischer Sympathie zu begleiten, zu beobachten und zu kommentieren. Ethnologie und Anthropologie haben uns offensichtlich etwas ,zu sagen“. ${ }^{16}$ 
https://doi.org/10.17104/9783406635991-95

Generiert durch IP '172.22.53.54', am 26.04.2023, 17:10:28.

Das Erstellen und Weitergeben von Kopien dieses PDFs ist nicht zulässig. 


\section{Begriffe und Theorien}

Die vorangegangenen Skizzen zur Fachgeschichte versuchten zu zeigen, wie sich bereits in den vorwissenschaftlichen Anfängen der Volkskunde bestimmte Leitbilder und Leitbegriffe herauskristallisieren, in denen sich ein sehr konkretes Erkenntnisinteresse widerspiegelt. Dieses Erkenntnisinteresse - und das ist entscheidend - wird zunächst in und von der Gesellschaft formuliert: Weltbilder, historische Zeitstimmungen, bestimmte politische, soziale und ethische Vorstellungen bilden den Rahmen einer Wissensordnung, die dann der Wissenschaft bestimmte Aufgaben und Fragestellungen nahelegt und die umgekehrt von deren Antworten und neuen Fragen mit geprägt wird. An jenem „Denken um das Volk“ zwischen französischer Aufklärung und deutscher Romantik wird dieser Mechanismus sehr deutlich. Im Umgang mit Begriffen wie „Volksseele“, „Volkslieder“ oder "Volkstum“ zeichnen sich dabei verschiedenartige Deutungsmöglichkeiten $\mathrm{ab}$ - etwa literarisch-ästhetische, zivilisatorisch-kulturelle oder politisch-nationale -, die ihren gemeinsamen Kern lediglich darin besitzen, daß sie ,Volk“ als Substrat von Kultur und Menschheit betrachten. Wie dann aus dieser allgemeinen Idee „Volk“ ein national praktiziertes „Volkstum“ werden kann, zeigt die Entwicklung zur „Deutschen Volkskunde“ in exemplarischer Weise. Daran läßt sich auch nachvollziehen, wie sehr die folgenden, sich wandelnden geschichtlichen und politischen Zeitstimmungen dann wieder Volksbilder und Volksbegriffe umprägen.

Für die Volkskunde ergibt sich aus diesen Leitbegriffen über lange Zeit kein eigenes Denk- und Erklärungsmodell. Ihre Vorstellungen von „Volk“ und Volkstum“ beschreiben kein wissenschaftliches Erkenntnisziel, sondern spiegeln lediglich eine vermeintliche Tatsache wider, die durch volkskundliches Sammeln und Dokumentieren allerdings wieder stärkere Realitätsmächtigkeit erhalten soll: Um sich zu erfüllen, muß „Deutsches Volkstum“ sich seiner selbst nur erneut bewußt werden. So verbleibt der Leitbegriff auf der Ebene einer Ideologie, die durch einen Kanon von Untersuchungsfeldern scheinbare Bodenhaftung erhält: in Vorstellungen von Tradition und Kontinuität, von Sitte und Brauch, von Märchen und Sage, von Lied und Dialekt, von Tracht und Speise, von Haus und Hof. Riehls Versuche, entlang der ,großen vier S“ von Stamm, Sprache, Sitte und Siedlung das System einer „Naturgeschichte des Volkes“ zu entwickeln, glie- 